\title{
A Novel Bimodal Imaging Agent Targeting HER2 Molecule of Breast Cancer
}

\author{
Wei Lv, ${ }^{1}$ Yamei Shen, ${ }^{1}$ Hengli Yang, ${ }^{1}$ Rui Yang, ${ }^{1}$ Wenbin Cai, ${ }^{1}$ Jian Zhang $\mathbb{D}^{2}{ }^{2}$ Lijun Yuan, \\ Yunyou Duan $\left(\mathbb{1},{ }^{1}\right.$ and Li Zhang $\left(\mathbb{1}^{1}\right.$ \\ ${ }^{1}$ Department of Ultrasound Diagnosis, Tang Du Hospital, Fourth Military Medical University, Xi'an, Shaanxi 710038, China \\ ${ }^{2}$ State Key Laboratory of Cancer Biology, Department of Biochemistry and Molecular Biology, Fourth Military Medical University, \\ Xi'an, Shaanxi 710032, China
}

Correspondence should be addressed to Yunyou Duan; duanyy@fmmu.edu.cn and Li Zhang; lilyzhang319_20@hotmail.com

Received 11 October 2017; Revised 24 December 2017; Accepted 2 January 2018; Published 6 March 2018

Academic Editor: Jian Song

Copyright ( $) 2018$ Wei Lv et al. This is an open access article distributed under the Creative Commons Attribution License, which permits unrestricted use, distribution, and reproduction in any medium, provided the original work is properly cited.

\begin{abstract}
Nanobubble (NB), a newly developed nanoscaled ultrasound contrast agent (UCA) for molecular imaging, has been widely researched for these years. Targeting it with functional molecule, nanobubble can adhere selectively to cellular epitopes and receptors outside the vasculature via enhanced permeability and retention (EPR) effect of tumor blood vessel. To enhance the targeting rate of our previous prepared NBs-Affibody for HER2 (+) breast cancer imaging, we introduced a near-infrared fluorescent (NIRF) dye, IR783, in this study to enhance tumor-specific targeting rate and provide a promising modality for dual-mode imaging. The prepared IR783-NBs-Affibody presented a uniform nanoscale size around $482.7 \pm 54.3 \mathrm{~nm}$, good biosecurity, and stability over time. The encapsulation efficiency (EE) of IR-783 was $15.09 \%$ in the conjugates leading to a successful NIR fluorescence and ultrasound enhancement imaging ex vivo. IR783-NBs-Affibody was able to automatically accumulate on BT474 cells with a highly increased targeting rate of $85.4 \%$ compared with previous NBs-Affibody of $26.6 \%$, while Affibody-guided HER2 binding was only found in HER2-positive cell lines (BT474 and T-47D). The newly developed IR783-NBs-Affibody is characterized with favorable HER2 targeting ability and bimodal imaging capability for breast cancer. Thus, IR783-NBs-Affibody holds great potential in molecular diagnosis for patients with breast cancer.
\end{abstract}

\section{Introduction}

The overexpressed HER2 receptor is a valuable biomarker for breast cancer as it accounts for about $15-20 \%$ for those patients who have been diagnosed with breast cancer. It is also the only one that has improved the clinical management for patients with HER2 $(+)$ by using anti-HER2 targeted agents, such as trastuzumab, lapatinib, and pertuzumab [1]. HER2 protein overexpression is measured by immunohistochemistry or by fluorescence in situ hybridization (FISH) test nowadays [2]. According to the published joint guideline recommendations in 2007 of the College of American Pathologists (CAP) and the American Society of Clinical Oncology (ASCO), it has been a routine pathological diagnosis to detect HER2 status in breast cancer all over the world $[3,4]$, not only for the prognostic evaluation for breast cancer patients but also a key element of the adjuvant therapy for HER2- positive patients. However, tumor heterogeneity may have been responsible for the false-negative finding in limited biopsy samples both in primary tumor and axillary lymph nodes (ALNs). Thus, a more convictive method for comprehensive assessment of HER2 status of breast cancer is needed.

Imaging modalities, especially, molecular imaging, may provide more precise in vivo information through a noninvasive approach, which would improve the understanding of pathological behavior of tumor at different therapy courses. Among all these imaging methods, ultrasound is well known for its nonradiation, time and cost saving, real-time imaging patterns, and so on. Microbubbles, or ultrasound contrast agent (UCA), such as SonoVue, applied as blood pool agents with microscaled diameters $[5,6]$ have been adapted for molecular imaging within blood vessels in many research works. These modified agents cannot leak outside the tumor vasculature to target a specific tumor-expressing molecule 
directly because of the agent size, which hampers the truly molecular imaging by ultrasound.

In our previous studies, we have fabricated a new nanosized UCA nanobubble (NB) through a revised thin-filmed hydration method [7] and targeted NB with a small HER2 antibody, Affibody [8]. The results demonstrated a high HER2-specific binding ability of NB-Affibody conjugates for HER2-positive breast cancer both ex vivo and in vivo. However, considering a limited targeting rate around 20\% and a comparatively poor ALN imaging capability for UCAs, in this study, we introduced another near-infra fluorescent dye as we tried previously [9], IR783, which has been approved for a tumor-targeting selectivity and enhanced new bubbles' targeting rate as well as a higher sensitivity for sentinel lymph node (SLN) mapping due to their low background autofluorescence. Besides, bimodal imaging modalities providing both optical and ultrasound imaging are complementary and will offer more diagnostic information for patients with breast cancer.

\section{Materials and Methods}

2.1. Materials. DPPC (phospholipids 1,2-dipalmitoyl-sn-glycero-3-phosphocholine; $\mathrm{Mw}=734 \mathrm{~g} / \mathrm{mol}$ ) and DSPE-PEGBiotin (2000) (1,2-distearoyl-sn-glycero-3-phosphoethanolamine- $\mathrm{N}$-[biotinylated(polyethyleneglycol)-2000]; $\mathrm{Mw}=$ $3017 \mathrm{~g} / \mathrm{mol}$ ) were used for preparing nanobubbles. Both were purchased from Avanti Polar Lipids Inc. (Alabaster, AL). Other materials were obtained as followed: IR783 from Sigma-Aldrich (St. Louis, MO), octafluoropropane (C3F8) gas from the R\&D Center (Beijing, China), ImmunoPure ${ }^{\circledR}$ streptavidin from Pierce (Rockford, IL), and biotinylated anti-ErbB2 Affibody ${ }^{\circledR}$ Molecule from Abcam (USA).

2.2. Preparation of the IR783-NBs-Affibody. In a dry $25 \mathrm{~mL}$ rotary evaporation bottle, $10 \mathrm{mg}$ DPPC and $4 \mathrm{mg}$ DSPEPEG-Biotin (2000) were mixed and $2 \mathrm{~mL}$ chloroform was added to dissolve the mixture. Then, $200 \mu \mathrm{L}$ NIRF agent IR783 liquor (dissolved in chloroform, $1 \mathrm{mg} / \mathrm{mL}$ ) was added. In a rotary evaporator (New Brunswick Scientific, New Brunswick, NJ), rotary evaporation was conducted at $120 \mathrm{rpm}$ and $55^{\circ} \mathrm{C}$. After 10 minutes, chloroform was completely evaporated and a uniform light green phospholipid thin film was developed. Subsequently, $1.5 \mathrm{~mL}$ hydration liquid (10\% glycerol and 90\% 1x PBS, $v / v)$ was used for the film hydration. The bottle was put into an incubatorshaker (New Brunswick Scientific, NJ) at $130 \mathrm{rpm}$ under $37^{\circ} \mathrm{C}$ for $60 \mathrm{~min}$, then the suspension of IR783-loaded liposomal film was prepared. The suspension was transferred into a tube. After the air in the tube was removed and $\mathrm{C}_{3} \mathrm{~F}_{8}$ gas was inflated, the tube was placed in a mechanical oscillator (Ag and $\mathrm{Hg}$ mixer, Xi'an, China) for $90 \mathrm{~s}$ to produce bubbles. To combine biotinylated anti-ErbB2 Affibody molecules with IR783-loaded NBs, the avidin-biotin method was applied. To prevent fluorescence quenching, tin foil was used to cover all bottles and tubes. In the control group, we used SonoVue. Finally, the IR783-NBs-Affibody was sterilized by $\mathrm{CO} 60$ irradiation for $30 \mathrm{~min}$.
2.3. Measurement of IR783-NBs-Affibody Characteristics. $1 \mathrm{x}$ PBS $(10 \mathrm{~mL})$ was used to dilute IR783-NBs-Affibody mother liquor $(1 \mathrm{~mL})$. The size distributions of IR783-NB-Affibody $(1.5 \mathrm{~mL})$ and SonoVue microbubble $(1.5 \mathrm{~mL})$ were measured by particle size analyzer (DelsaNano, Beckman Coulter, USA) at $25^{\circ} \mathrm{C}$. The results were calculated as a mean value after three repeated measurements.

2.4. Morphologic Observation of IR783-NBs-Affibody. To further investigate the IR783-NBs-Affibody structure, transmission electron microscopy (TEM, FEIT12, America) was applied. SonoVue was also examined as control. A copper grid covered with amorphous carbon was used to dry the samples for $30 \mathrm{~min}$ before observing.

2.5. Efficiency of Encapsulation (EE) and Loading Rate of IR783 in IR783-NBs. The amount of the IR783 in IR783NBs was determined by standard curve of UV-vis absorption spectra measured by a UV-vis spectrophotometer (Mapada UV-6100S, Shanghai, China). IR783-NBs was separated from the original solution by centrifugation of $1500 \mathrm{rpm}$ for $5 \mathrm{~min}$ at $4^{\circ} \mathrm{C}$. All the agents, IR783, DPPC, DSPE-PEGBiotin, and IR783-NBs, were dissolved in ethyl alcohol. The encapsulation efficiency and loading rate of IR783 were calculated as follows:

$$
\begin{aligned}
\mathrm{EE}(\%)= & \frac{\text { weight of IR783 in IR783 }-\mathrm{NBs}}{\text { weight of total added IR783 }} \\
& \times 100 \%, \\
\text { IR783 loading rate }(\%)= & \frac{\text { weight of IR783 in IR783 - NBs }}{\text { weight of IR783 - NBs }} \\
& \times 100 \% .
\end{aligned}
$$

2.6. Biosecurity of IR783-NBs-Affibody. BT-474 human breast cancer cells were cultured in Dulbecco's modified Eagle's medium (DMEM) with 10\% fetal bovine serum (FBS) and placed in a humidified atmosphere of $5 \% \mathrm{CO}_{2}$ at $37^{\circ} \mathrm{C}$. Then, BT- 474 cells were seeded at a density of 5000 cells/well in 96-well plate. The cells were cultured for $24 \mathrm{~h}$ till they adhered to bottom. Subsequently, the cells were cultured with various concentrations of IR783 $(1-40 \mu \mathrm{g} / \mathrm{mL})$ in fresh medium for $24 \mathrm{~h}$. Then $100 \mu \mathrm{L}$ of fresh medium with $10 \mu \mathrm{L}$ CCK- 8 was added to culture these cells for $4 \mathrm{~h}$. The absorbance at $450 \mathrm{~nm}$ of each well was measured after the plate was oscillated at low speed for $30 \mathrm{~s}$ in a Microplate Reader (iMark, Bio-Rad, America).

2.7. NIRF and Ultrasound Bimodal Imaging of IR783-NBsAffibody In Vitro. Optical imaging capability of IR783-NBsAffibody was observed by laser scanning confocal microscopy (LSCM, Nikon A1R, Japan) with excitation at $640 \mathrm{~nm}$ and peak emission at $780 \mathrm{~nm}$ to obtain NIR fluorescent.

To complete ultrasound contrast imaging, a latex glove fingertip filling with $10 \mathrm{~mL}$ of $1 \mathrm{x}$ PBS was put in water at $25^{\circ} \mathrm{C}$. MyLab Twice ultrasound system (Esaote, Italy) was used to acquire ultrasound images with a $5 \mathrm{MHz}$ transducer. After the ultrasound images of PBS-containing glove can be 


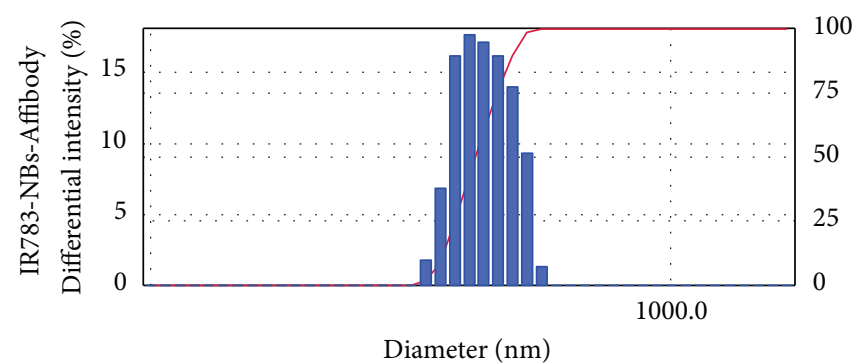

(a)

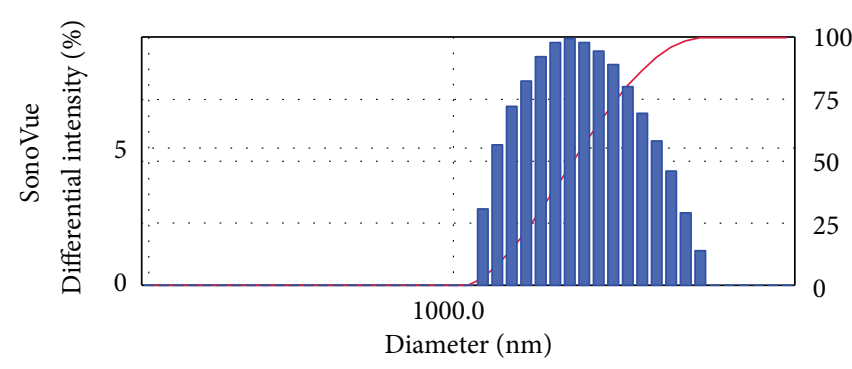

(b)

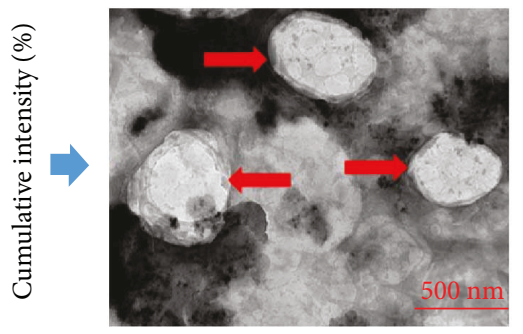

(c)

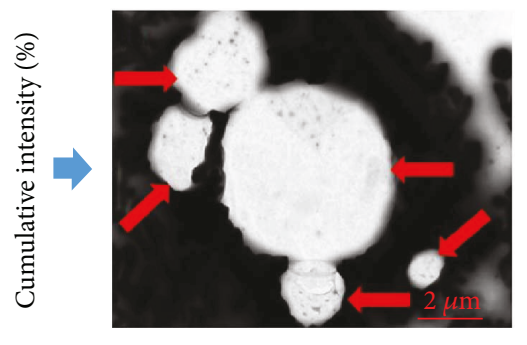

(d)

Figure 1: Comparisons of IR783-NanoBubble-Affibody and SonoVue in size distribution and morphology. Size distribution of IR783-NBsAffibody (a) and SonoVue microbubbles (b). TEM images of IR783-NBs-Affibody (c) and SonoVue microbubbles (d).

acquired clearly, SonoVue and IR783-NBs-Affibody $(100 \mu \mathrm{L})$ were injected into PBS, respectively, then the ultrasound pictures were compared.

2.8. Stability of IR783-NBs-Affibody. A $10 \mu \mathrm{L}$ solution of IR783-NBs-Affibody was dissolved in $1 \mathrm{~mL} 1 \mathrm{x}$ PBS in a tube at $37^{\circ} \mathrm{C}$. The change of the IR783-NBs-Affibody in the size distribution was measured for $20 \mathrm{~min}$ at every 5 min interval using the particle size analyzer. The diluted IR783-NBs-Affibody was exposed at $37^{\circ} \mathrm{C}$ for $20 \mathrm{~min}$, and hemocytometer was used to calculate the number at 1,5 , 10,15 , and $20 \mathrm{~min}$. All the abovementioned measurements were performed in triplicate.

2.9. Tumor-Specific Targeting of IR783-NBs-Affibody In Vitro. Three breast cancer cell lines (HER2-positive cells BT-474 and T-47D and HER2-negative cell MDA-MB-231) were incubated in the same condition as previously mentioned. When the BT- 474 cells grew up to $70-80 \%$ confluence, all cells were trypsinized, divided into sterile four tubes (marked 1-4) with the same cell number, and centrifuged to remove culture medium. PBS, NBs, NBs-Affibody, and IR783-NBs-Affibody were added into tubes 1 to 4 , respectively, at room temperature for $0.5 \mathrm{~h}$. Then, the cells were washed with PBS for three times by centrifugation (Beckman Coulter, Fullerton, CA) before flow cytometry (FCM) analysis, the same operations for MDA-MB-231.

In a $20 \mathrm{~mm}$ confocal dish, after the BT-474 and MDAMB-231 cells were attached to the bottom, the medium was discarded and the cell was washed with sterile $1 \mathrm{x}$ PBS lightly. NBs, NBs-Affibody, and IR783-NBs-Affibody $\left(200 \mu \mathrm{L}, 6.0 \times 10^{6} / \mathrm{mL}\right)$ were added to the dish, respectively, and coincubated at room temperature for $0.5 \mathrm{~h}$. After being washed with PBS for three times, the cells were incubated with $4 \%$ formaldehyde $(1 \mathrm{~mL})$ for $15 \mathrm{~min}$ and kept at $4^{\circ} \mathrm{C}$, then washed for three times. Subsequently, $0.1 \%$ Triton X$100(1 \mathrm{~mL})$ was added to enhance the penetration of substrate at room temperature for $10 \mathrm{~min}$ and washed away. Next, $1 \mu \mathrm{g} / \mathrm{mL}$ DAPI $\left(4^{\prime}, 6\right.$-diamidino-2-phenylindole) was added for nucleus staining and after 5-minute incubation, it was washed with $1 \mathrm{x}$ PBS. Fluorescence images were captured by LSCM with a $\times 20$ objective lens. Each fluorescent was obtained at different wavelengths, for example, DAPI blue fluorescent was obtained at $405 \mathrm{~nm}$ for excitation and $475 \mathrm{~nm}$ for emission, $\mathrm{DiO}$ green fluorescent was obtained at $488 \mathrm{~nm}$ for excitation and $530 \mathrm{~nm}$ for emission, and IR783 NIR fluorescent was obtained at $640 \mathrm{~nm}$ for excitation and $780 \mathrm{~nm}$ for emission. HER2 targeting ability of IR783NBs-Affibody was further assessed with FCM by both $\mathrm{DiO}$ and IR783 fluorescence detection in HER2 (+)/HER2 $(-)$ cell lines. All the above procedures were conducted in dark three times.

\section{Results}

3.1. Size Distribution and Morphology of IR783-NBs-Affibody. The mean diameters of IR783-NBs-Affibody and SonoVue microbubbles were $482.7 \pm 54.3 \mathrm{~nm}$ and $1784.7 \pm$ $400.4 \mathrm{~nm}$, respectively. The polydispersity index (P.I.) was $0.3107 \pm 0.068(n=3)$ and $0.280 \pm 0.036(n=3)$ accordingly (Figures 1(a) and 1(b)).

IR783-NBs-Affibody and SonoVue microbubbles obviously appeared spherical under TEM scanning. The size of SonoVue was over $1000 \mathrm{~nm}$, while IR783-NBs-Affibody showed to be around $500 \mathrm{~nm}$ (Figures 1(c) and 1(d)). The results of particle size analyzer and TEM were consistent. 


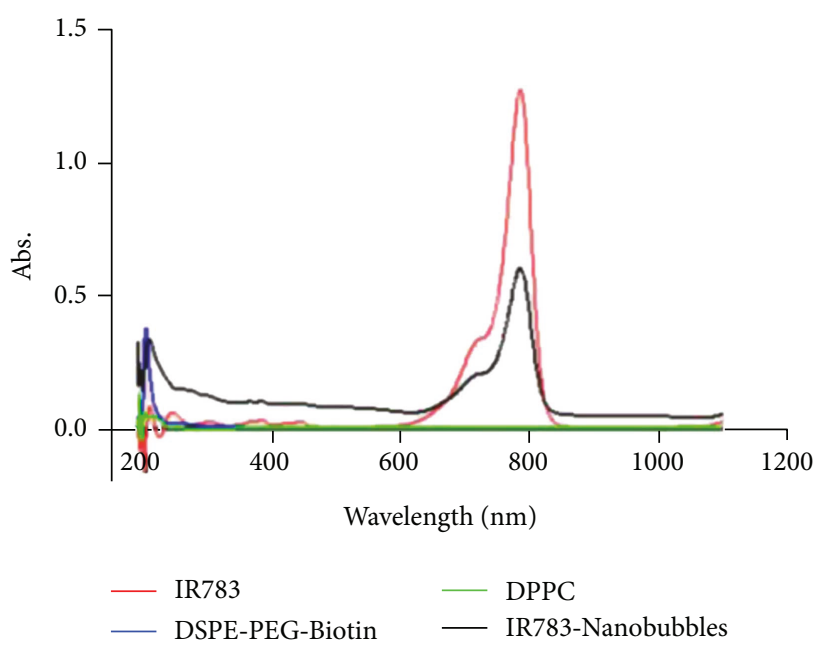

(a)

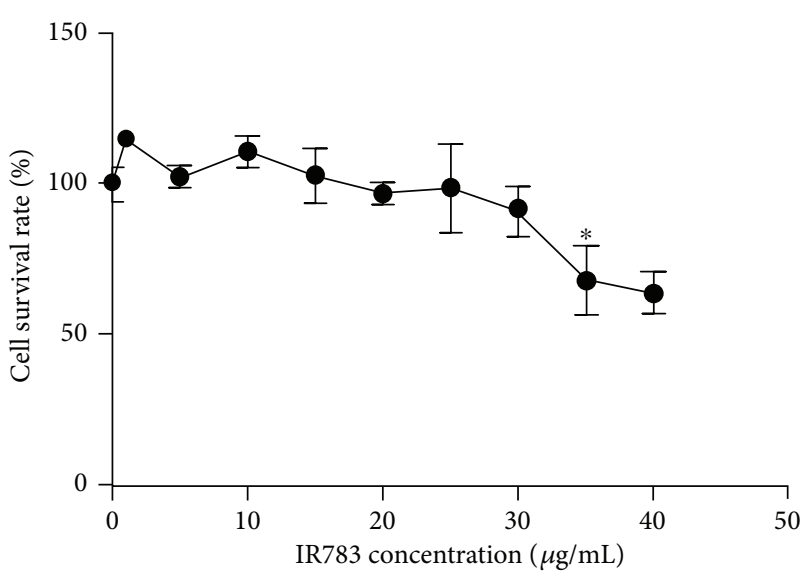

(b)

FIGURE 2: IR783-loaded NBs (IR783-NBs) and its biosecurity. (a) Absorbance spectra were measured in IR783, DPPC, DSPE-PEG-Biotin, and IR783-NBs in ethyl alcohol. The adsorption peak of $200 \mathrm{~nm}$ wavelength is caused by DSPE-PEG-Biotin and DPPC. (b) Cytotoxicity of different concentrations of IR-783 in BT-474 cells, as examined by a CCK- 8 assay.

3.2. IR783 Loaded in IR783-NBs and Its Biosecurity. The EE of IR783 in IR783-NBs was about $15.09 \%$, and loading rate was $0.21 \%$. IR783 $(3 \mu \mathrm{g} / \mathrm{mL}$ in ethyl alcohol) exhibited a strong absorption peak at $786 \mathrm{~nm}$, while DPPC and DSPEPEG-Biotin showed absorption about $200 \mathrm{~nm}$ wavelength and no absorption in near-infrared region from UV-vis spectra. After being loaded with IR783, IR783-NBs appeared to have the same absorption peak at $786 \mathrm{~nm}$ as IR783 does without shift (Figure 2(a)).

Cell survival rate curve was obtained via cell counting kit (CCK-8) assay (Figure 2(b)). Obvious cytotoxicity was not observed until IR783's concentration was increased up to $35 \mu \mathrm{g} / \mathrm{mL}$, indicating that IR783 had no obvious cytotoxicity to BT-474 cells at a normal concentration $(<5 \mu \mathrm{g} / \mathrm{mL})$ for research and clinical purposes.

3.3. Bimodal Imaging of IR783-NBs-Affibody In Vitro. Fluorescence inspection of IR783-NBs-Affibody was performed under LSCM scanning. As shown in Figure 3(a), many bubbles smaller than $1 \mu \mathrm{m}$ were clearly observed against a dark-field background and IR783 fluorescence was detected on the nanoscaled bubbles.

Compared with the negative control, IR783-NBsAffibody exhibited significantly enhanced ultrasonic contrast ability as SonoVue MBs did (Figure 3(b)).

3.4. The Stability of IR783-NBs-Affibody. The stability of IR783-NBs-Affibody was evaluated by two physical parameters including size distribution and concentration. The size distribution of IR783-NBs-Affibody was not significantly changed after 15 min compared to that of IR783-NBsAffibody at $1 \mathrm{~min}(627.4 \pm 125.5 \mathrm{~nm}$ versus $461.5 \pm 113.2 \mathrm{~nm}$, $P>0.05$; Figure 4(a)). Nevertheless, the IR783-NBs-Affibody at $20 \mathrm{~min}$ changed into bigger bubbles obviously different from the ones at $1 \mathrm{~min}(811.0 \pm 234.4 \mathrm{~nm}$ versus $461.5 \pm$
113.2 nm, $P<0.05$; Figure 4(a)). Similarly, the concentration of IR783-NBs-Affibody was kept stable at $15 \mathrm{~min}$ compared with that at $1 \mathrm{~min}\left(12.3 \pm 0.4 \times 10^{6} / \mathrm{mL}\right.$ versus $11.5 \pm$ $0.8 \times 10^{6} / \mathrm{mL}, P>0.05$; Figure $\left.4(\mathrm{~b})\right)$, while the concentration of IR783-NBs-Affibody was significantly decreased after 20 minutes $\left(9.3 \pm 0.6 \times 10^{6} / \mathrm{mL}\right.$ at $20 \mathrm{~min}$ versus $11.5 \pm$ $0.8 \times 10^{6} / \mathrm{mL}$ at $1 \mathrm{~min}, P<0.05$, Figure $\left.4(\mathrm{~b})\right)$.

3.5. The Tumor-Specific Targeting of IR783-NBs-Affibody. The tumor-specific targeting rate towards HER2 (+) breast tumor cells BT-474 was increased greatly after IR-783 was introduced in the bubble from $26.6 \%$ of NBs-Affibody to $85.4 \%$ of IR783-NBs-Affibody (Figure 5(a)). For HER2 (-) breast tumor cells MDA-MB-231, the tumor-specific targeting rate was also increased from $5.4 \%$ of NBs-Affibody to 99.3\% of IR783-NBs-Affibody (Figure 5(c)). LSCM images also confirmed that an accumulation of NBs-Affibody fluorescence on BT-474 cells was observed compared with NBs (Figure 5(b)) and with NBs-Affibody towards MDA-MB231 without green fluorescence (Figure 5(d)). NIR fluorescence from IR783-NBs-Affibody was shown around almost all cells, and the amount of fluorescence accumulation in IR783-NBs-Affibody was much higher than that of NBsAffibody in both BT-474 and MDA-MB-231 cells.

Further fluorescence detection demonstrated that only IR783-NBs-Affibody showed both tumor-specific and HER2-specific binding abilities towards HER2 (+) tumor cells. The HER2 targeting rate of IR783-NBs-Affibody was kept at $19.4 \%$ and $17.3 \%$ for BT-474 and T-47D cells, respectively (Figure 6).

\section{Discussion}

The major achievement of this study is to prepare a new nanobubble that can be served as a bimodal imaging agent 


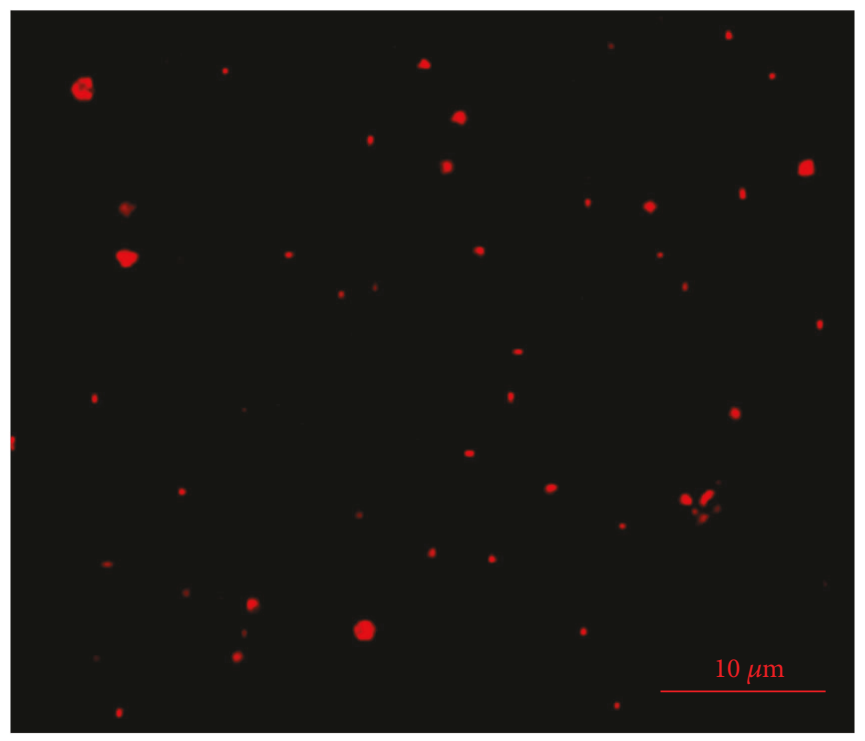

(a)

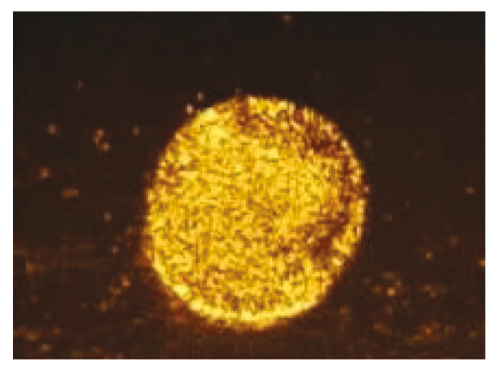

IR783-NBs-Affibody

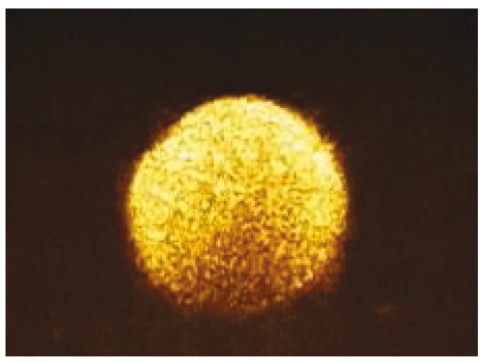

SonoVue

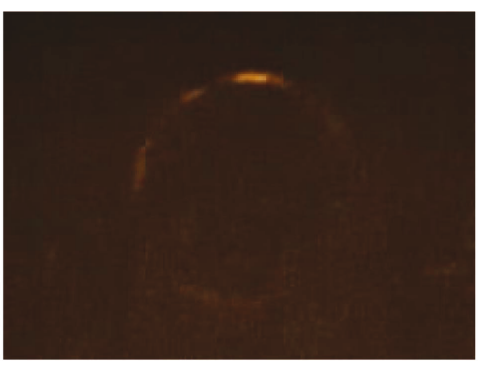

PBS

(b)

FIGURE 3: NIRF and ultrasound bimodal images of IR783-NBs-Affibody in vitro. (a) A LSCM image of IR783-NBs-Affibody. (b) The contrastenhanced ultrasound images of IR783-NBs-Affibody, SonoVue microbubbles, and PBS in vitro, respectively.

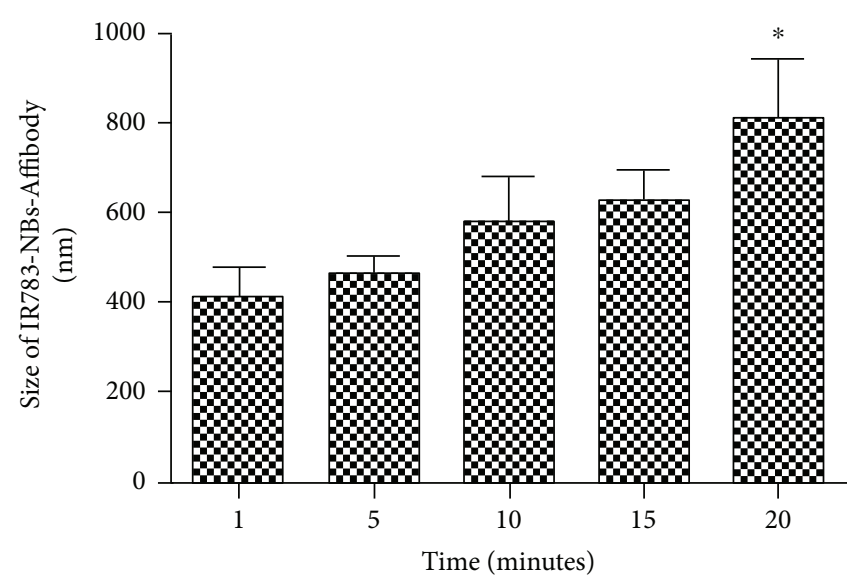

(a)

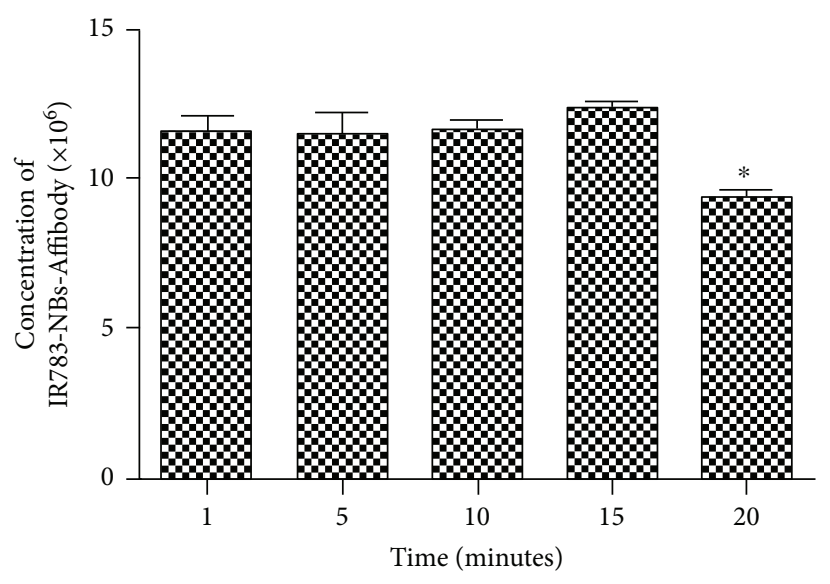

(b)

FIGURE 4: Stability of IR783-NBs-Affibody. (a) The changes of IR783-NBs-Affibody's size distribution over 20 min. ${ }^{*} P<0.05$, obviously different from ones at $1 \mathrm{~min}$. (b) The changes of IR783-NBs-Affibody's concentration over 20 min. ${ }^{*} P<0.05$, obviously different from ones at $1 \mathrm{~min}$.

in both contrast-enhanced ultrasound (CEUS) and optical imaging for patients with breast cancer to identify both primary tumor and small metastasis and also determine the expression status of HER2 within tumor. As we know, the majority of patients can be diagnosed with breast cancer at early stage with the development of modern imaging 

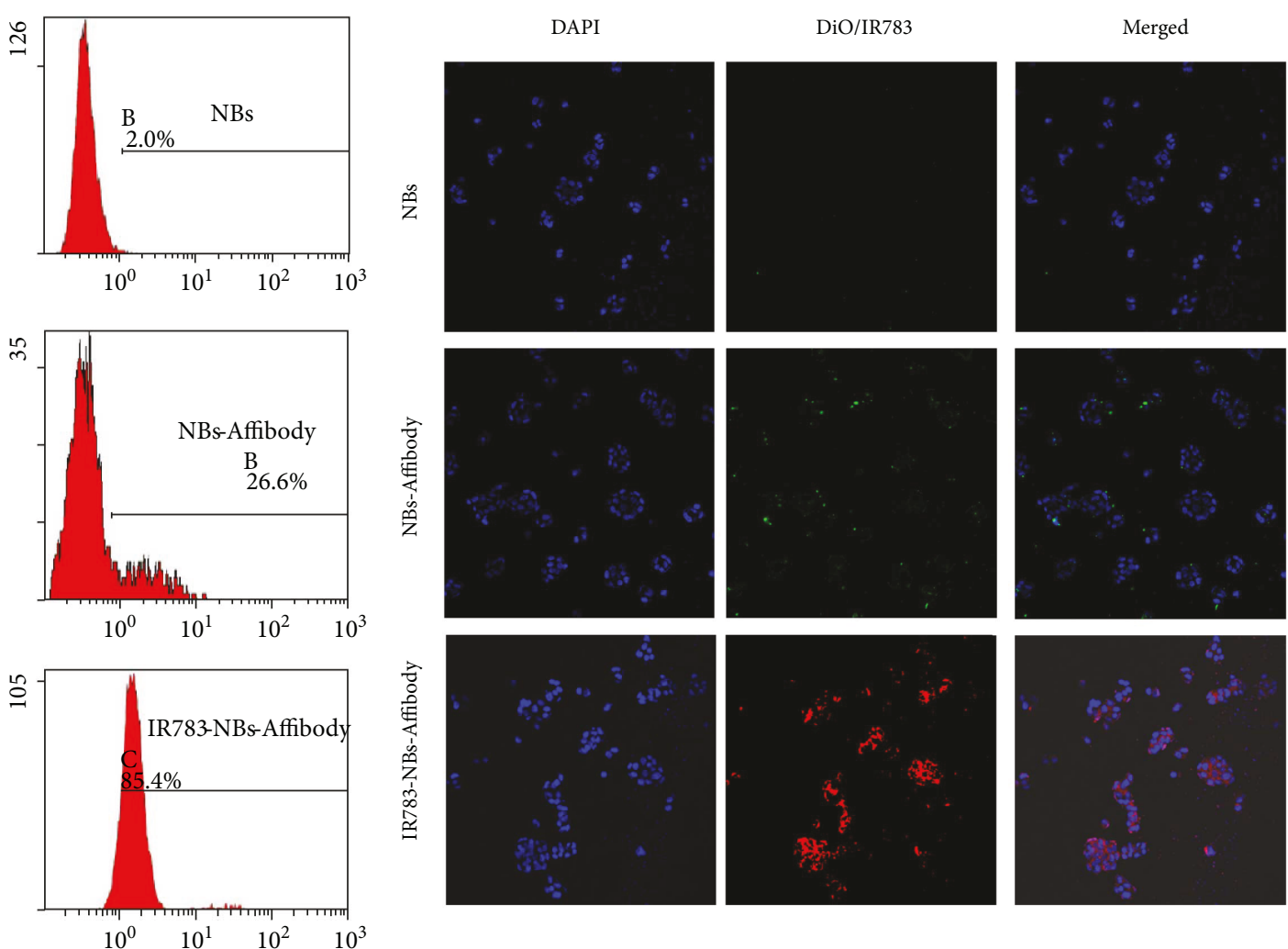

(a)
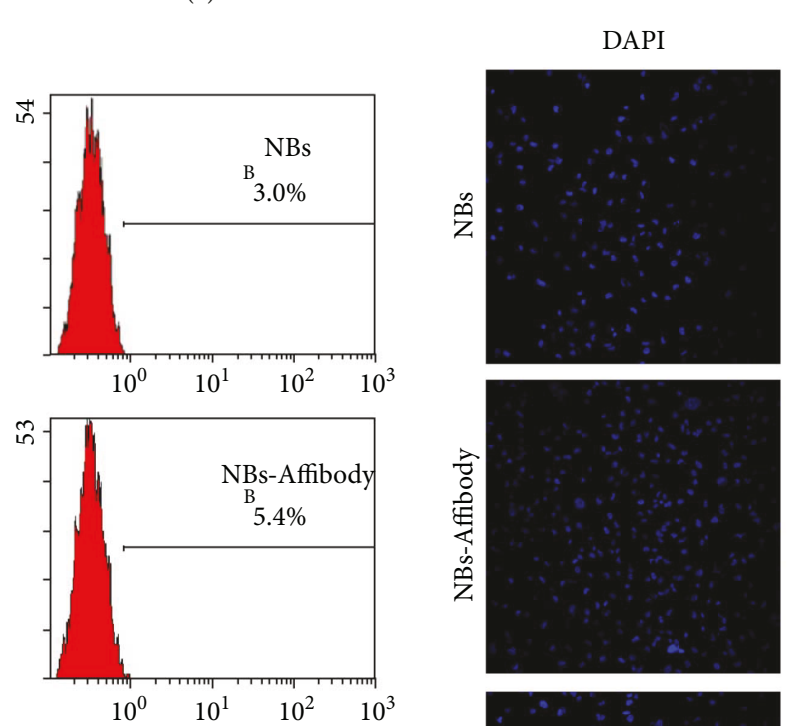

(b)
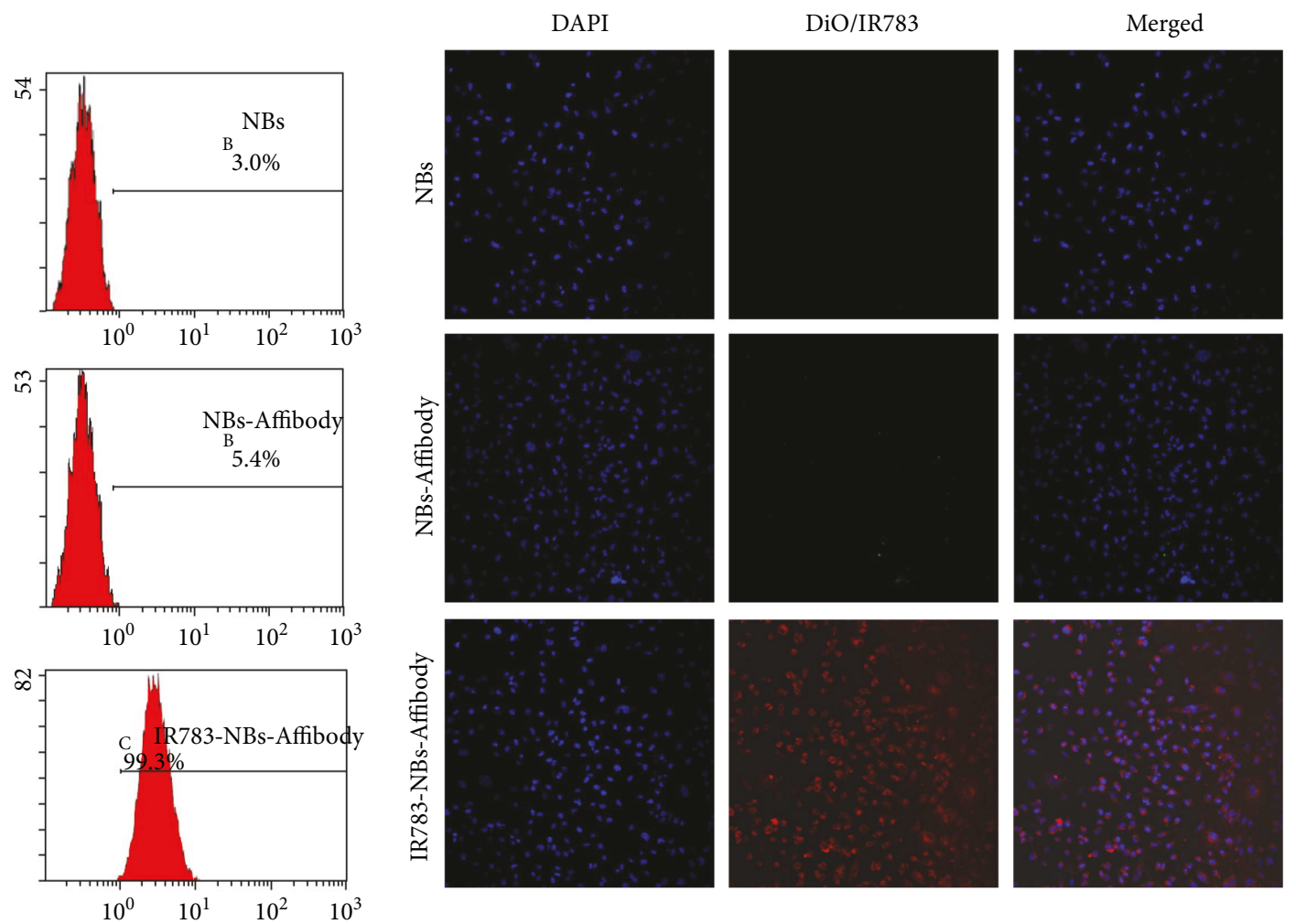

(c)

(d)

FIGURE 5: The tumor-specific targeting of IR783-NBs-Affibody towards HER2 (+)/(-) breast cancer cells. (a, c)The targeting rate of NBs, NBsAffibody, and IR783-NBs-Affibody for HER2 (+) breast cancer cells (BT-474) and HER2 (-) breast cancer cells (MDA-MB-231) via flow cytometry. (b, d) LSCM pictures of BT-474 and MDA-MB-231 cells coincubated with NBs, NBs-Affibody, and IR783-NBs-Affibody with a $\times 20$ objective lens. 

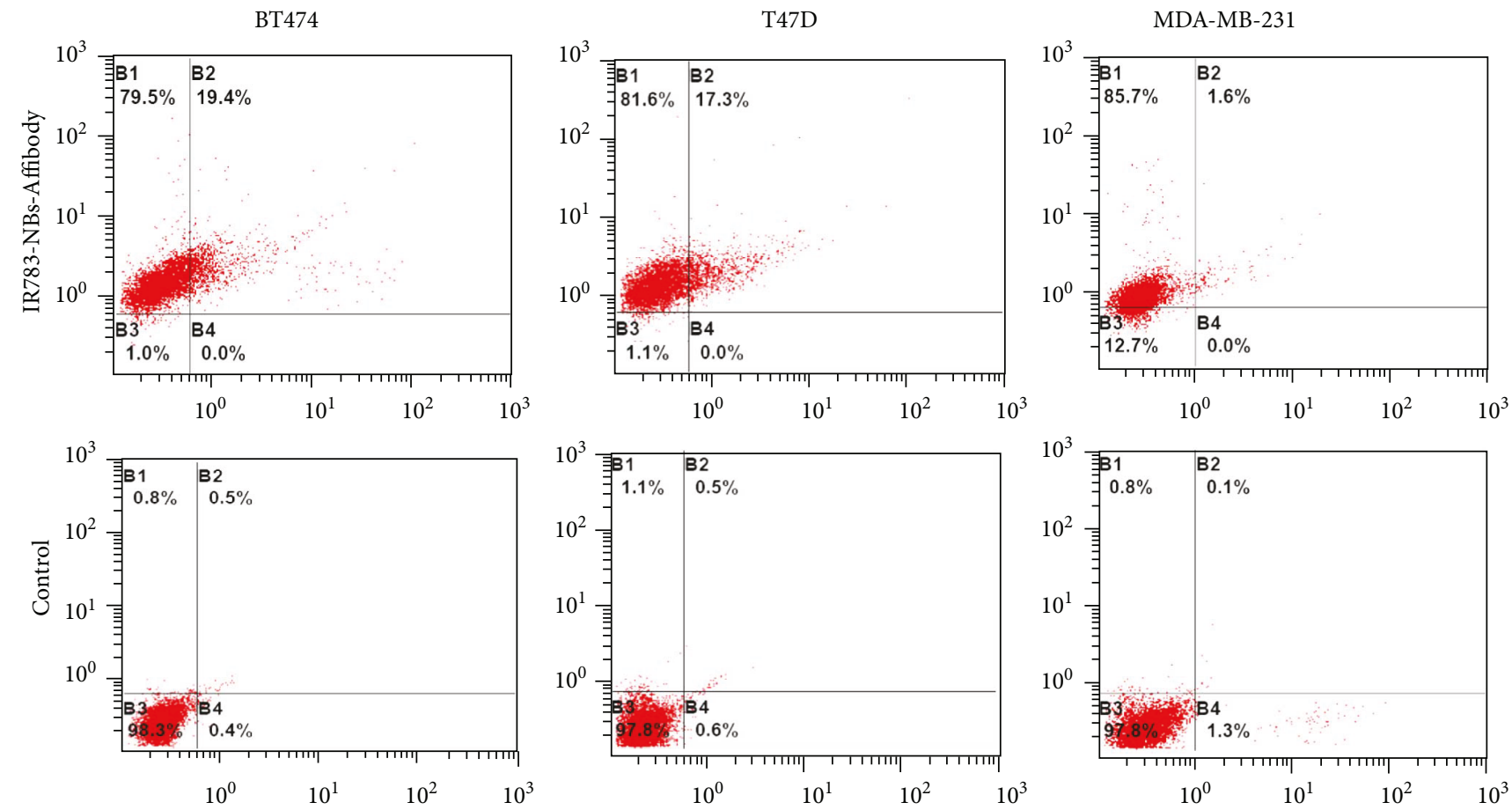

FIGURE 6: The HER2-specific targeting rate of IR783-NBs-Affibody towards HER2 (+) breast cancer cells BT-474/T-47D and HER2 (-) breast cells MDA-MB-231. The B1 area represents cells dyed with IR783, B2 for cells dyed with both IR783 and DiO, B3 for cells without fluorescence, and $\mathrm{B} 4$ for cells dyed with $\mathrm{DiO}$.

technologies nowadays. For those patients, breast conserving therapy $(\mathrm{BCT})$ is recommended $[10,11]$. Identification of surgical margin and ALN involvements may enhance clinical preoperative decision-making for BCT. Although intraoperative ultrasound (IOUS) and CEUS further increase the capability of localizing the surgical margin and potential ALNs, many questions still remain. Without tumor or tumor subtype molecule targeting, US and CEUS are incapable of judging the malignant margin or nodes from benign ones [12-14]. In previous studies, HER2-targeting nanobubbles showed promise in HER2 targeting ability both in vitro and in vivo [8]. However, the HER2 status varies between a primary and metastatic site [15]. A total of $13 \%$ of cancers that were HER2 $(+)$ in primary cancer were found to be HER2 (-) in metastatic lesion, whereas $5 \%$ to be negative in the primary tumor were positive in metastatic specimen in a meta-analysis [16]. Tumor heterogeneity may have been responsible for the negative finding in the biopsy sample. Thus, an over 20\% HER2-binding rate of NBsAffibody that has been observed may not be sufficient for a promising signal-to-noise ratio (SNR) imaging, which depends on the contrast agent's biodistribution to acquire a signal strength comparison between the targeted tissue and the surrounding area.

IR783, as well as IR-780 iodide and MHI-148, has been demonstrated with preferential accumulation in cancer tissues by itself with no need of conjugation to guiding moieties $[17,18]$. Despite of the unclear mechanism of the cyanine dyes' specific accumulation and retention, some researches have illustrated that the implications of EPR effect, organic-anion transporting polypeptides (OATPs), and mitochondrial membrane potential might have some relation $[19,20]$. However, because of the existing lipophilic profile, NIRF dyes are extremely difficult to dissolve in water, which hampers their clinical utilization greatly. Nowadays, interests have been proposed in improving the biocompatibility of NIR fluorescence dyes by reengineering them with different carriers for cancer diagnosis and therapy $[21,22]$. Among these carriers, phospholipid-shell and gascore NBs exhibited ideal compatibility in encapsulating NIR dyes [6,23-25]. Our study confirmed a successful conjugation of IR783 with NBs with the EE about 15.09\% through simple loading instead of chemical conjugation. The EE which was low might be the reason of the processing procedure that IR783-NBs was separated from the original solution by centrifugation of $1500 \mathrm{rpm}$ for $5 \mathrm{~min}$ at $4^{\circ} \mathrm{C}$. It is difficult to obtain all IR783-NBs after centrifugation because the stability of IR783-NBs would be challenged by bubble-burst and some IR783-NBs became adhesive to the wall. Although the EE and loading rate of IR783 were low, IR783-NBs-Affibody presented optimal tumor targeting towards HER2 $(+) /(-)$ breast cancer cells via FCM and LSCM tests.

As our results indicated that by introducing IR783, an amphiphilic molecule with NIRF imaging and tumortargeting capabilities into previous NBs-Affibody, the newly prepared IR783-NBs-Affibody, showed a much higher tumor-specific binding ability to BT474 cells and MDAMB-231 higher than $80 \%$ compared with previous NBsAffibody. NIR fluorescence was accumulated around most 
of the breast cancer cells as detected by LSCM in IR783-NBsAffibody group. The passive targeting through EPR effect, HER2 binding ligand, and IR783 tumor auto-selectivity was enhanced greatly. The targeting rate of IR783-NBs-Affibody was found to be similar either to HER2-positive (BT-474 and T-47D) or to HER2-negative (MDA-MB-231) cells. HER2-labelled fluorescent cells were detected only in HER2-positive cell lines. The reason might be that IR783mediated tumor binding is a kind of tissue-specific targeting in differentiating tumor tissue from normal tissue, but not a specific molecule binding for further tumor subtype imaging, even without the tumor subtype recognition unit. Thus, by integrating anti-HER2 monoclonal antibody and heptamethine dye IR783 into the nanoscaled UCA, the IR783 function of IR783-NBs-Affibody enables the tumor-specific recognition for primary tumor and possible metastasis, while the Affibody function determines the HER2 expression. More interestingly, the binding rate of IR783-NBsAffibody to HER2-positive tumor cells varies depending on the variability of HER2 expression on different cell lines. According to Chen et al.'s report that BT474 cells express HER2 higher than T-47D cells do [26], we detected more HER2-labelled cell group in the present study. This finding can be applied in quantitative diagnosis of HER2 expression level in further studies.

Imaging with high spatial resolution is urgently required for accurate tumor margin delineation and localization of potential small metastases. Thus, bimodal or multimodal imaging agent has attracted increasing attention as it can provide a multitechnical complementation to achieve more imaging information about the lesion and also offer a potential agent-mediated strategy [27-29]. On the one hand, both imaging agents can be easily conjugated together in the preparation procedure and also showed favorable physical properties including uniform nanoscale size, good stability, and low cytotoxicity. On the other hand, the dual-modality imaging of optical and ultrasound would provide a complementary mode for tumor molecular diagnosis. Several studies reported that modified NIR fluorescence by conjugating various molecules showed excellent accuracy in the intraoperative detection of tumor margins [30, 31]. NIR fluorescence is also a good tracer in visualizing the lymphatics and lymph nodes [32-34] and has been explored for intraoperative image-guiding modality $[34,35]$ with diverse degrees of success. The preferential accumulation of NIR fluorescence in tumor can provide a significantly higher SNR in tumors $[36,37]$. There are still drawbacks in optical imaging method because of the absorbing and scattering features of bio-tissue and rapid optical fluorescence declining with the depth increasing. Nevertheless, the ultrasonic scattering of UCA could help imaging agents to achieve high acoustic resolution at depths. Our result also indicated that IR783-NBs-Affibody showed similar echogenic ability with SonoVue in vitro because the compressible liposome membrane is apt to form and yield echogenicity allowing a high scattering of NBs under a low MI and harmonic imaging modes [38, 39]. Therefore, CEUS is compatible with optical imaging, thereby achieving bimodality imaging by a complementary contrast.

\section{Conclusion}

The obtained bimodal imaging agent IR783-NBs-Affibody providing both ultrasound and near-infrared fluorescence imaging signals is able to achieve tumor-specific and HER2specific targeting simultaneously. IR783-NBs-Affibody presents nanoscale uniform size, a low polydispersity, and favorable stability. For ultrasound mode, IR783-NBs-Affibody shows a promising enhanced ultrasound signal in vitro similar to SonoVue does; while for NIR fluorescence mode, IR783-NBs-Affibody-treated tumor cells exhibit high fluorescence intensity. Thus, IR783-NBs-Affibody is expected to achieve a high SNR when two imaging modalities are applied in breast cancer patients, which is helpful for tumor delineation and possible SNL trace.

\section{Conflicts of Interest}

The authors declare that there is no conflict of interest regarding the publication of this article.

\section{Authors' Contributions}

Wei Lv performed the laboratory assays and wrote the manuscript. Yamei Shen helped the laboratory experiments and interpret the data. Hengli Yang and Rui Yang were assigned in the data acquisition. Wenbin Cai performed the statistical analysis. Jian Zhang and Lijun Yuan helped in interpreting the laboratory results. Yunyou Duan reviewed and approved the final version of the manuscript. Li Zhang designed this study, revised, and approved the final version of the manuscript. All authors have given the final approval of the version to be published. Wei Lv and Yamei Shen are co-first authors to this work.

\section{Acknowledgments}

This study was financially supported by the National Natural Science Foundation of China (Grant no. 81571730). The authors are grateful to the Department of Molecular Biology and the Department of Pharmaceutical Analysis, Fourth Military Medical University, for their technical support and kind provision of equipment.

\section{References}

[1] D. J. Slamon, B. Leyland-Jones, S. Shak et al., "Use of chemotherapy plus a monoclonal antibody against HER2 for metastatic breast cancer that overexpresses HER2," New England Journal of Medicine, vol. 344, no. 11, pp. 783-792, 2001.

[2] A. C. Wolff, M. E. Hammond, D. G. Hicks et al., "Recommendations for human epidermal growth factor receptor 2 testing in breast cancer: American Society of Clinical Oncology/ College of American Pathologists clinical practice guideline update," Journal of Clinical Oncology, vol. 31, no. 31, pp. 3997-4013, 2013.

[3] D. Slamon, W. Eiermann, N. Robert et al., "Adjuvant trastuzumab in HER2-positive breast cancer," New England Journal of Medicine, vol. 365, no. 14, pp. 1273-1283, 2011. 
[4] J. H. Ha, M. K. Seong, E. K. Kim et al., "Serial serum HER2 measurements for the detection of breast cancer recurrence in HER2-positive patients," Journal of Breast Cancer, vol. 17, no. 1, pp. 33-39, 2014.

[5] A. T. Nguyen and S. P. Wrenn, "Acoustically active liposomenanobubble complexes for enhanced ultrasonic imaging and ultrasound-triggered drug delivery," Wiley Interdisciplinary Reviews: Nanomedicine and Nanobiotechnology, vol. 6, no. 3, pp. 316-325, 2014.

[6] K. W. Ferrara, M. A. Borden, and H. Zhang, "Lipid-shelled vehicles: engineering for ultrasound molecular imaging and drug delivery," Accounts of Chemical Research, vol. 42, no. 7, pp. 881-892, 2009.

[7] W. B. Cai, H. L. Yang, J. Zhang et al., "The optimized fabrication of nanobubbles as ultrasound contrast agents for tumor imaging," Scientific Reports, vol. 5, no. 1, article 13725, 2015.

[8] H. Yang, W. Cai, L. Xu et al., "Nanobubble-Affibody: novel ultrasound contrast agents for targeted molecular ultrasound imaging of tumor," Biomaterials, vol. 37, pp. 279-288, 2015.

[9] H. Yang, T. Zhou, W. Cai et al., "Novel dual-mode nanobubbles as potential targeted contrast agents for female tumors exploration," Tumor Biology, vol. 37, no. 10, pp. 1415314163, 2016.

[10] A. Goldhirsch, J. N. Ingle, R. D. Gelber et al., "Thresholds for therapies: highlights of the St Gallen international expert consensus on the primary therapy of early breast cancer 2009," Annals of Oncology, vol. 20, no. 8, pp. 1319-1329, 2009.

[11] S. L. Blair, K. Thompson, J. Rococco, V. Malcarne, P. D. Beitsch, and D. W. Ollila, "Attaining negative margins in breast-conservation operations: is there a consensus among breast surgeons?," Journal of the American College of Surgeons, vol. 209, no. 5, pp. 608-613, 2009.

[12] P. D. Britton, A. Goud, S. Godward et al., "Use of ultrasoundguided axillary node core biopsy in staging of early breast cancer," European Radiology, vol. 19, no. 3, pp. 561-569, 2009.

[13] S. Rautiainen, M. Sudah, S. Joukainen, R. Sironen, R. Vanninen, and A. Sutela, "Contrast-enhanced ultrasound -guided axillary lymph node core biopsy: diagnostic accuracy in preoperative staging of invasive breast cancer," European Journal of Radiology, vol. 84, no. 11, pp. 21302136, 2015.

[14] H. Eggemann, T. Ignatov, S. D. Costa, and A. Ignatov, “Accuracy of ultrasound-guided breast-conserving surgery in the determination of adequate surgical margins," Breast Cancer Research and Treatment, vol. 145, no. 1, pp. 129-136, 2014.

[15] M. J. Duffy, N. Harbeck, M. Nap et al., "Clinical use of biomarkers in breast cancer: updated guidelines from the European group on tumor markers (EGTM)," European Journal of Cancer, vol. 75, pp. 284-298, 2017.

[16] G. Aurilio, D. Disalvatore, G. Pruneri et al., "A meta-analysis of oestrogen receptor, progesterone receptor and human epidermal growth factor receptor 2 discordance between primary breast cancer and metastases," European Journal of Cancer, vol. 50, no. 2, pp. 277-289, 2014.

[17] X. Yang, C. Shi, R. Tong et al., "Near IR heptamethine cyanine dye-mediated cancer imaging," Clinical Cancer Research, vol. 16, no. 10, pp. 2833-2844, 2010.

[18] X. Yi, F. Wang, W. Qin, X. Yang, and J. Yuan, "Near-infrared fluorescent probes in cancer imaging and therapy: an emerging field," International Journal of Nanomedicine, vol. 9, pp. 13471365, 2014.
[19] C. Shi, J. B. Wu, G. C. Chu et al., "Heptamethine carbocyanine dye-mediated near-infrared imaging of canine and human cancers through the HIF-1 $\alpha$ /OATPs signaling axis," Oncotarget, vol. 5, no. 20, pp. 10114-10126, 2014.

[20] J. Yuan, X. Yi, F. Yan et al., "Nearinfrared fluorescence imaging of prostate cancer using heptamethine carbocyanine dyes," Molecular Medicine Reports, vol. 11, no. 2, pp. 821-828, 2014.

[21] C. Jiang, H. Cheng, A. Yuan, X. Tang, J. Wu, and Y. Hu, "Hydrophobic IR780 encapsulated in biodegradable human serum albumin nanoparticles for photothermal and photodynamic therapy," Acta Biomaterialia, vol. 14, pp. 61-69, 2015.

[22] S. Lee, R. George Thomas, M. Ju Moon et al., "Near-infrared heptamethine cyanine based iron oxide nanoparticles for tumor targeted multimodal imaging and photothermal therapy," Scientific Reports, vol. 7, no. 1, p. 2108, 2017.

[23] J. Liu, B. Zhang, M. Li et al., "Preparation and characterization of a novel silicon-modified nanobubble," PLoS One, vol. 12, no. 5, article e0178031, 2017.

[24] X. Fan, Y. Guo, L. Wang, X. Xiong, L. Zhu, and K. Fang, "Diagnosis of prostate cancer using anti-PSMA aptamer A10-3.2oriented lipid nanobubbles," International Journal of Nanomedicine, vol. Volume 11, pp. 3939-3950, 2016.

[25] Y. Gao, C. Hernandez, H. X. Yuan et al., "Ultrasound molecular imaging of ovarian cancer with CA-125 targeted nanobubble contrast agents," Nanomedicine, vol. 13, no. 7, pp. 2159-2168, 2017.

[26] Y. Chen, J. Qian, Q. He et al., "mTOR complex-2 stimulates acetyl-CoA and de novo lipogenesis through ATP citrate lyase in HER2/PIK3CA-hyperactive breast cancer," Oncotarget, vol. 7, no. 18, pp. 25224-25240, 2016.

[27] L. Zhang, H. Zhou, O. Belzile, P. Thorpe, and D. Zhao, "Phosphatidylserine-targeted bimodal liposomal nanoparticles for in vivo imaging of breast cancer in mice," Journal of Controlled Release, vol. 183, pp. 114-123, 2014.

[28] B. Luo, H. Zhang, X. Liu, R. Rao, Y. Wu, and W. Liu, "Novel DiR and SPIO nanoparticles embedded PEG-PLGA nanobubbles as a multimodalimaging contrast agent," Bio-medical Materials and Engineering, vol. 26, no. s1, pp. S911-S916, 2015.

[29] R. R. Kudarha and K. K. Sawant, "Albumin based versatile multifunctional nanocarriers for cancer therapy: fabrication, surface modification, multimodal therapeutics and imaging approaches," Materials Science and Engineering: C, vol. 81, pp. 607-626, 2017.

[30] J. S. Mieog, M. Hutteman, J. R. van der Vorst et al., "Imageguided tumor resection using real-time near-infrared fluorescence in a syngeneic rat model of primary breast cancer," Breast Cancer Research and Treatment, vol. 128, no. 3, pp. 679-689, 2011.

[31] S. A. Suganya, K. J. Kochurani, M. G. Nair et al., "TM1-IR680 peptide for assessment of surgical margin and lymph node metastasis in murine orthotopic model of oral cancer," Scientific Reports, vol. 6, no. 1, article 36726, 2016.

[32] T. Ikeda, T. Sugie, A. Shimizu, and M. Toi, "Patterns of clinical practice for sentinel lymph node biopsy in women with nodenegative breast cancer: the results of a national survey in Japan," Breast Cancer, vol. 24, no. 2, pp. 341-344, 2017.

[33] J. Li, B. Jiang, C. Lin, and Z. Zhuang, "Fluorescence tomographic imaging of sentinel lymph node using near-infrared emitting bioreducible dextran nanogels," International Journal of Nanomedicine, vol. 9, pp. 5667-5682, 2014. 
[34] W. Guo, L. Zhang, J. Ji, W. Gao, J. Liu, and M. Tong, "Breast cancer sentinel lymph node mapping using near-infrared guided indocyanine green in comparison with blue dye," Tumor Biology, vol. 35, no. 4, pp. 3073-3078, 2014.

[35] H. S. Choi, S. L. Gibbs, J. H. Lee et al., "Targeted zwitterionic near-infrared fluorophores for improved optical imaging," Nature Biotechnology, vol. 31, no. 2, pp. 148-153, 2013.

[36] S. Luo, E. Zhang, Y. Su, T. Cheng, and C. Shi, "A review of NIR dyes in cancer targeting and imaging," Biomaterials, vol. 32, no. 29, pp. 7127-7138, 2011.

[37] X. Tan, S. Luo, D. Wang, Y. Su, T. Cheng, and C. Shi, “A NIR heptamethine dye with intrinsic cancer targeting, imaging and photosensitizing properties," Biomaterials, vol. 33, no. 7, pp. 2230-2239, 2012

[38] K. Ferrara, R. Pollard, and M. Borden, "Ultrasound microbubble contrast agents: fundamentals and application to gene and drug delivery," Annual Review of Biomedical Engineering, vol. 9, no. 1, pp. 415-447, 2007.

[39] M. B. Flegg, C. M. Poole, A. K. Whittaker, I. Keen, and C. M. Langton, "Rayleigh theory of ultrasound scattering applied to liquid-filled contrast nanoparticles," Physics in Medicine and Biology, vol. 55, no. 11, pp. 3061-3076, 2010. 


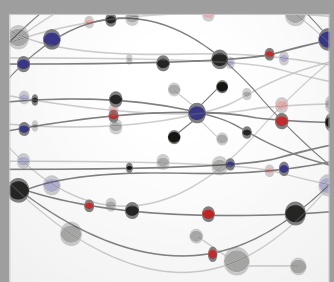

The Scientific World Journal
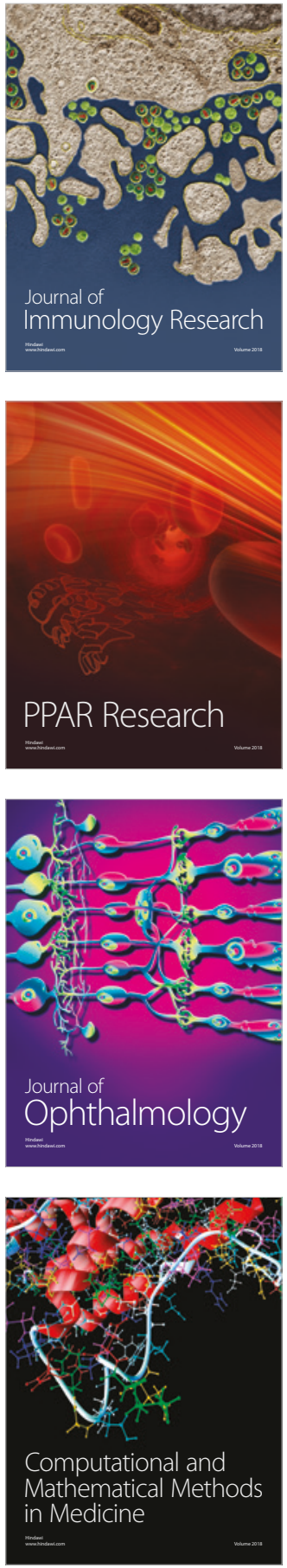

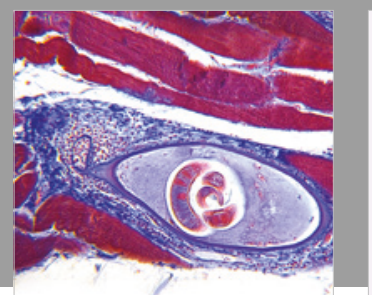

Gastroenterology Research and Practice

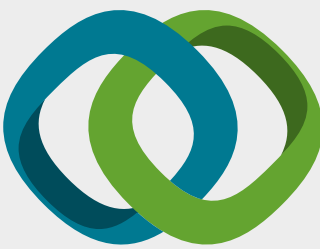

\section{Hindawi}

Submit your manuscripts at

www.hindawi.com
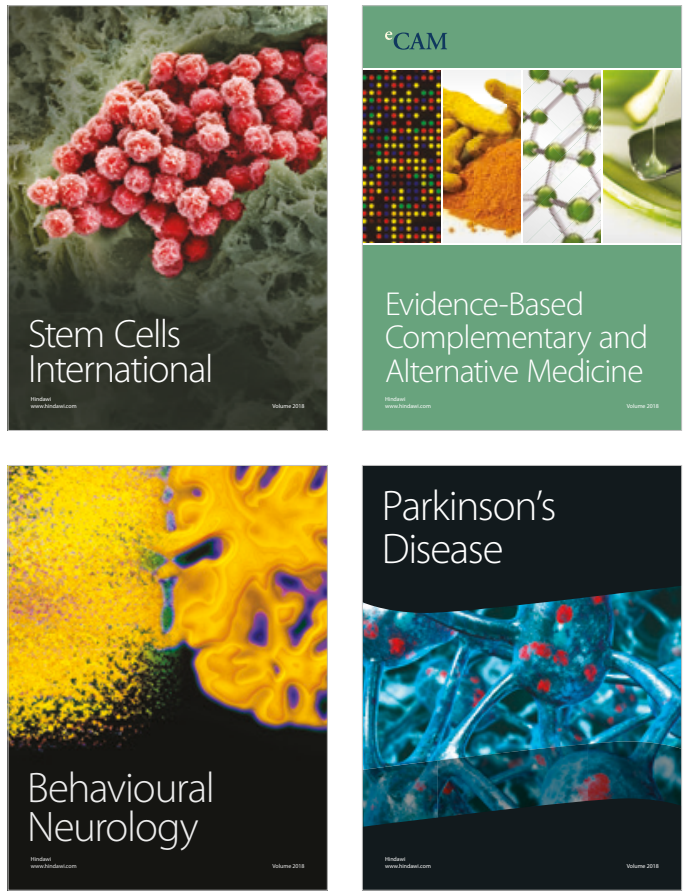

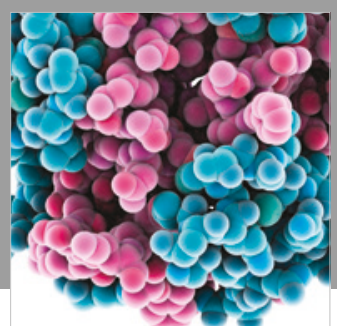

ournal of

Diabetes Research

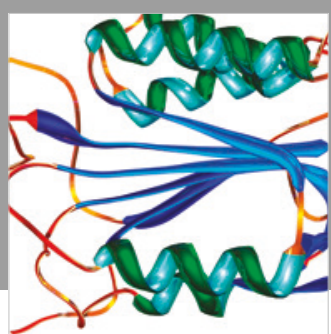

Disease Markers
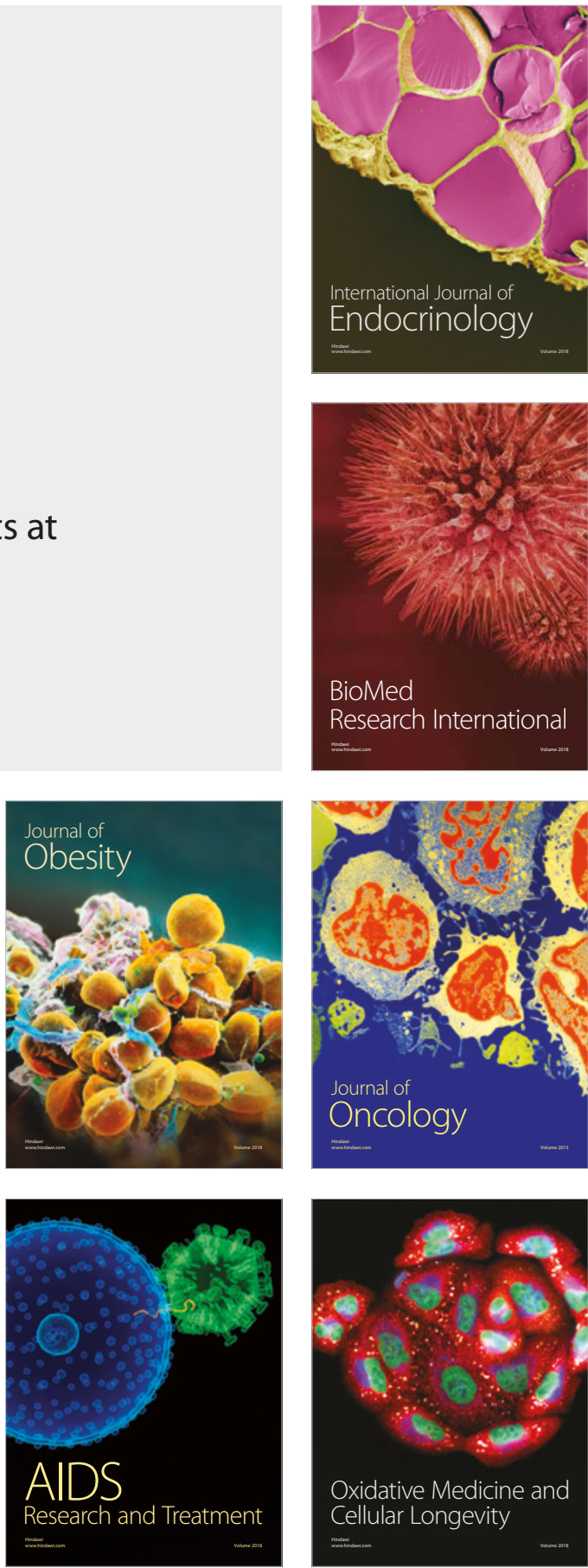